\title{
The role of relativistic kinematics in describing two-quark systems
}

\author{
V.Lengyel ${ }^{1}$, V.Rubish ${ }^{1}$, Yu.Fekete ${ }^{1}$, S.Chalupka ${ }^{2}$, M.Salak ${ }^{3}$ \\ 1 Department of Theoretical Physics, Uzhgorod State University, \\ 32 Voloshin St., UA-294000 Uzhgorod, Ukraine \\ 2 Department of Theoretical Physics and Geophysics, P.J.Safarik University, \\ 16 Moyzesova St., 04154 Košice, Slovak Republic \\ 3 Department of Physics, Prešov University, \\ 17 Novembra St., 08009 Prešov, Slovak Republic
}

Received February 17, 1998

An attempt to incorporate relativistic kinematics in the description of light quark systems is made. It seems that the way of such an incorporation suggested by R.Gaida and his collaborators is very promising. Comparison of these results with the experimental data concerning a boson mass spectrum shows that this approach is among the best theoretical interpretations of the data.

Key words: light quarks, potential model, Dirac equation, mesons

PACS: $05.20 .-y, 03.30 .+p$

Surprising success of non-relativistic quark models with a funnel or oscillator potential in describing hadron mass spectra for heavy quark systems has inspired the hope that at least some aspects of strong interactions are finally understood. A good insight into the previous works concerning this subject is given in [1-3]. But evidently the picture is not complete until it incorporates the light quarks $\mathrm{u}, \mathrm{d}$ and s. But this means that the problem is shifting into a quantum chromodynamical sector. In this respect a search for the alternative possibilities of incorporating relativistic kinematics is quite actual. A very promising approach to the solution of the relativistic problem of interaction of two particles was recently suggested by R.Gaida and his collaborators [4-7]. Their results can be directly applied to our problem of calculating the mass spectrum of quarkonium as a system of two quarks. A somewhat different approach was simultaneously developed by I.Todorov and P.Bogolyubov [8-9], and later by E.Predazzi et al. [10]. In this work we shall investigate the results concerning the application of their findings to light-quark systems. 
First, let us follow the way suggested by Predazzi et al. [10-12]. Following the ideas expressed in [10,11] and later developed in [12], let us start with the classical expression for the relativistic total energy of a two-particle system with masses $m$ and $M$, respectively,

$$
E=\sqrt{\mathbf{p}_{1}^{2}+m^{2}}+\sqrt{\mathbf{p}_{2}^{2}+M^{2}},
$$

were $\mathbf{p}_{1}=-\mathbf{p}_{2} \equiv \mathbf{p}$ in the c.m. system. The main problem here is the mode of inclusion of the interaction between the two particles. On the basis of (1) and passing to quantum mechanics, Lucha and Schoberl [13] write for equal masses

$$
\left(2 \sqrt{\mathbf{p}^{2}+m^{2}}+V\right) \Psi(\mathbf{r})=E \Psi(\mathbf{r})
$$

which they call the spinless Bethe-Salpeter equation. They cunningly solve this equation in the configurational momentum representation but do not obtain very good results which we shall discuss later. But in any case we can state that it is the way of introducing the interaction which is responsible for their failure. Therefore, we propose here another way of introducing the interaction. Namely, following Predazzi et al. [12] we linearize the expression

$$
\left(\frac{E^{2}+m^{2}-M^{2}}{2 E}\right)^{2}=\mathbf{p}^{2}+m^{2}
$$

which follows from (1) after simple algebraic transformations and obtain

$$
\left(\frac{E^{2}+m^{2}-M^{2}}{2 E}\right) \Psi(\mathbf{r})=\left(\alpha c \mathbf{p}+\beta m c^{2}+\widetilde{V}\right) \Psi(\mathbf{r}),
$$

where $\alpha$ and $\beta$ are the usual Dirac matrices, $\Psi$ is a four-component wave function for which we shall use the two-component representation $\Psi(\mathbf{r})=\left(\begin{array}{c}\varphi(\mathbf{r}) \\ \chi(\mathbf{r})\end{array}\right)$. In the general case we would have to decide on this stage what kind of Lorentz-transform properties we shall ascribe to the interaction.

In general, the interaction can transform either like a Lorentz-scalar (like mass) or be a 4 th component of a Lorentz-vector, i.e. transform like energy. Then we shall consider the interaction $\widetilde{V}$ as a mixture

$$
\widetilde{V}=\beta S \cdot(1-\varepsilon)+\mathbf{I} V \cdot \varepsilon,
$$

where $\varepsilon$ is some mixing parameter. In what follows we shall simplify the expression for $\widetilde{V}$ by taking $\varepsilon=1 / 2$ (which is suggested by the experimental data (see e. g. [14] and [15]) which means that (5) can be written in the form:

$$
\widetilde{V}=\frac{1}{2}(\beta+\mathbf{I})(V+S)
$$

This form is chosen in order to obtain the simple nonrelativistic result $\widetilde{V}=V$ if there is no difference between $S$ and $V$. Such a potential was introduced previously 
by V.Kukulin, M.Moshinsky et al. $[16,17]$ and was named an averaged potential. It allows one to reduce the system of Dirac equations to a single relativistic oscillator equation. In this particular case, when the scalar part $S$ and the vector part $V$ of potentials are equal, we shall consider the following possibilities:

$$
S+V \equiv \tilde{V}=\left\{\begin{array}{lr}
-\frac{\alpha_{\mathrm{s}}}{r_{\mathrm{s}}}+A \cdot r^{2}+V_{0} & \text { (7a) } \\
-\frac{\alpha_{\mathrm{s}}}{r}+\mathrm{k} \cdot r+V_{0} & (7 \mathrm{~b}) \\
\frac{g^{2}}{6 \pi \mu}\left(1-\mathrm{e}^{-\mu r}\right)-\frac{16 \pi}{25} \frac{\mathrm{e}^{-k r}}{r \cdot \ln \left(b+\left(\frac{1}{\Lambda r}\right)^{2}\right)}+V_{0} \cdot & \text { (7c) }
\end{array}\right\}
$$

Today all of these potentials are used to describe the quark-antiquark interaction. The discussion concerning the advantages and handicaps of these potentials is given in [18]. Our aim is to apply these potentials to describing the meson mass spectra with relativistic kinematics which is built into equation (4). In comparison with [18], a search for the best description by minimizing $\chi^{2}$ will be presented. For an averaged potential (6) the equation (4) reduces to a single equation for the "large" wave function $\varphi(\mathbf{r})$

$$
(E-V)^{2} \varphi(\mathbf{r})=\left[4 \mathbf{p}^{2}+4 m^{2}+4 m S+S^{2}\right] \varphi(\mathbf{r})=\left[4 \mathbf{p}^{2}+4\left(m+\frac{S}{2}\right)^{2}\right] \varphi(\mathbf{r}) .
$$

For this purpose we have to solve numerically the following equation:

$$
\left[\mathbf{p}^{2}+\left(\frac{E}{2}+m\right) \cdot \frac{\tilde{V}}{2}-\left(\frac{E^{2}}{4}-m^{2}\right)\right] \varphi(\mathbf{r})=0 .
$$

Passing to operators and carrying out substitutions for the unknown function $\varphi(\mathbf{r})=\Phi(\mathbf{r}) / \mathbf{r}$, one obtains the equation

$$
\left[\frac{\mathrm{d}^{2}}{\mathrm{~d} r^{2}}-\frac{l(l+1)}{r^{2}}-\left(\frac{E}{2}+m\right) \cdot \frac{\tilde{V}}{2}+\frac{E^{2}}{4}-m^{2}\right] \Phi(\mathbf{r})=0 .
$$

In the simple approximation $S+V=\frac{1}{2}\left(A r^{2}+V_{0}\right)$ one obtains

$$
\left[\mathbf{p}^{2}+\left(\frac{E}{2}+m\right)\left(\frac{1}{2} A r^{2}+\frac{1}{2} V_{0}\right)-\left(\frac{E^{2}}{4}-m^{2}\right)\right] \varphi(\mathbf{r})=0,
$$

which leads to the equation for a relativistic isotropic oscillator

$$
\left[\frac{\mathrm{d}^{2}}{\mathrm{~d} r^{2}}-\frac{l(l+1)}{r^{2}}-\left(\frac{E}{2}+m\right) \cdot \frac{1}{2} A r^{2}+\frac{E^{2}}{4}-m^{2}-\left(\frac{E}{2}+m\right) \cdot \frac{1}{2} V_{0}\right] \Phi(\mathbf{r})=0 .
$$

Now with the standard change of variables $\sqrt{\left(\frac{E}{2}+m\right) \frac{1}{2} A} \cdot r^{2}=x^{2}$ one obtains

$$
\left[\frac{\mathrm{d}^{2}}{\mathrm{~d} x^{2}}-\frac{l(l+1)}{x^{2}}-x^{2}+\frac{\frac{E^{2}}{4}-m^{2}-\frac{1}{2} V_{0}\left(\frac{E}{2}+m\right)}{\sqrt{\left(\frac{E}{2}+m\right) \frac{A}{2}}}\right] \Phi(\mathbf{r})=0 .
$$


The physical solution of (6) is satisfied when

$$
\frac{E^{2}}{4}-m^{2}=\sqrt{\frac{A}{2} \cdot \frac{E+2 m}{2}} \cdot(4 N+2 l+3)+\frac{V_{0}(E / 2+m)}{2}
$$

or

$$
\frac{E}{2}-m=\sqrt{\frac{A}{E+2 m}}(4 N+2 l+3)+\frac{V_{0}}{2} .
$$

In the nonrelativistic limit, when $E \sim 2 m$, one has

$$
E \approx 2 m+\sqrt{\frac{A}{m}}(4 N+2 l+3)+V_{0}
$$

in full accordance with the nonrelativistic case (see e.g. [18]).

Actually, for large total energies $E$, from (15) it follows that $E^{2} \sim l^{4 / 3}$ i.e. almost a linear Regge-trajectory as it should follow from general considerations. As we shall see, the application of other variants of the potentials gives even better results.

As we mentioned above, a very promising approach to the problem of the relativistic description of a many-particle system was elaborated along with the ideas presented by Gaida in [4] and elaborated by Tretyak, Shpytko and Duviryak [5-7]. They used the Weyl quantization method and succeeded in solving the problem for a relativistic oscillator between two particles.

Considering oscillator-type interaction

$$
V=\frac{1}{2} \omega^{2} \mathbf{p}_{1} \mathbf{p}_{2} r^{2}
$$

where $\mathbf{p}_{1} \mathbf{p}_{2}=m_{\mathrm{RED}}=m_{q} / 2$, they obtain the nonrelativistic approximation for the mass of a two-particle system

$$
M=\left\{\left[2 m_{q}+\frac{\omega}{2}(4 N+2 l+3)\right]^{2}+\frac{\omega^{2}}{4}\right\}^{1 / 2}+V_{0},
$$

or

$$
M=\sqrt{\left(\sum m_{q}+\sqrt{\frac{A}{m_{q}}} \cdot(4 N+2 l+3)\right)^{2}+\frac{A}{m_{q}}}+V_{0}
$$

if we express (17) in the form of a string tension $A$ and generalize their results to our boundary condition of an isotropic oscillator.

The same approximation was taken in [18]. But, if we suggest that in general

$$
\mathbf{p}_{1} \mathbf{p}_{2}=\frac{E+m_{q}}{4}
$$

then the Lviv group's result

$$
M=\left\{\left[2 m_{q}+\sqrt{\frac{2 A}{E+m_{q}}} \cdot(4 N+2 l+3)\right]^{2}+\frac{2 A}{E+m_{q}}\right\}^{1 / 2}+V_{0}
$$


will resemble very much our relativistic approach. The results of calculations according to (18) are given below. The parameters are taken to be $\mathrm{A}=0.01 \mathrm{GeV}^{3}$, $V_{0}=-0.436 \mathrm{GeV}$. Certainly, this approximation is valid if one considers the energy dependence of $\mathbf{p}_{i}$ only at the final stage of the calculations.

It is interesting to note that the similar to (18) result for $\mathbf{M}^{2}$-operator was obtained in the relativistic approach by Ishida-Oda based on a special assumption of the covariant relativistic approach. Even the numerical values of their parameters [19] are close to the result of (18). Their value $A=0.05 \mathrm{GeV}^{3}$ is of the same order as ours. A more precise comparison is impossible because of the ambiguity of other parameters.

Usually such potential models, like the one we used here, are called naive quark models. But our model is not so naive. Firstly, the relativistic kinematics not only renders it more complicated, but shows a possible way of building the model of interaction of two relativistic particles. Secondly, the potentials like (7) incorporate asymptotic freedom, the strong coupling constant $\alpha_{\mathrm{S}}$ is calculated according to the "classical" expression

$$
\alpha_{\mathrm{S}}(r)=\frac{12 \pi}{33-2 N} \cdot\left[\ln \left(\frac{1}{r^{2} \widetilde{\Lambda}^{2}}\right)\right]^{-1},
$$

where $\widetilde{\Lambda}$ is taken to be equal to $\widetilde{\Lambda}=0.14 \mathrm{GeV}$. And, finally, the model allows us to include a spin-spin interaction either by passing to the Breit-Fermi equation or by using the Dirac equation straightly. In table $1 \alpha_{\mathrm{s}}$ is taken exactly according to (19).

It is interesting to note that the definition of mass can be given in a different way. Considering one particle as moving in the field of another, and vice versa, and adding the obtained masses we obtain the results with the parameters which correspond very closely to our table 1 . Namely, one can write down the Dirac equation for one particle moving in the outer field, reduce it to the equation for the "large" component $\chi(\mathbf{r})$ and obtain [20]:

$$
\left(E^{2}-m^{2}\right) \chi(\mathbf{r})=\mathbf{p}^{2} \chi(\mathbf{r})+(E+m) V \chi(\mathbf{r}) .
$$

Applying the virial theorem to this equation,

$$
\left\langle\chi(y)\left|\mathbf{p}^{2}(y)\right| \chi(y)\right\rangle=\frac{1}{2}\left\langle\chi(y)\left|y \frac{\partial V}{\partial y}\right| \chi(y)\right\rangle=\left\langle\chi(y)\left|y^{2}\right| \chi(y)\right\rangle .
$$

Combining (20) and (21) for the oscillator interaction one obtains

$$
E^{2}-m^{2}=2 \sqrt{C(E+m)} B_{N l}+(E+m) V_{0}
$$

where

$$
B_{N l}=\left\langle\chi(y)\left|y^{2}\right| \chi(y)\right\rangle=2 N+l+3 / 2
$$

This expression resembles very much (18). So we have five most realistic from our point of view possibilities of calculating masses according to (7a)-(7c), (17), (18). 
To obtain the masses of a multi-quark system according to these expressions, it is necessary to define the values of the parameters. The masses of quarks were taken to be $m_{\mathrm{u}}=0.33 \mathrm{GeV}, m_{\mathrm{c}}=1.675-1.75 \mathrm{GeV}, m_{\mathrm{b}}=5.05-5.1 \mathrm{GeV}$, as usual in quark models. Parameters for different potentials are shown in table 1.

Table 1. The parameter values for different potentials.

\begin{tabular}{rcccll}
\hline Potential & $\alpha_{\mathrm{u}}$ & $\alpha_{\mathrm{c}}$ & $\alpha_{\mathrm{b}}$ & $k, A$ & $V_{0}, \mathrm{GeV}$ \\
\hline$(7 a)$ & 0.5 & 0.325 & 0.3 & $k=0.27 \mathrm{GeV}^{2}$ & -0.8356 \\
$(7 b)$ & 0.5 & 0.386 & 0.3 & $A=0 \ldots \mathrm{GeV}^{3}$ & -0.527 \\
$(7 c)$ & $\frac{g^{2}}{6 \pi}=0.3795 \mathrm{GeV}^{2}$ & $\mu=0.054 \mathrm{GeV}$ & $K=0.75 \mathrm{GeV}$ & $\Lambda=0.35 \mathrm{GeV}, b=4$ & -1.103 \\
\hline
\end{tabular}

Table 2. Variation of $\chi^{2}$ for different parameters of potential (7b).

\begin{tabular}{lrrrl}
\hline$k$ & $\chi_{\mathrm{u} \overline{\mathrm{u}}}^{2}$ & $\chi_{\mathrm{c} \overline{\mathrm{c}}}^{2}$ & $\chi_{\mathrm{b} \overline{\mathrm{b}}}^{2}$ & $V_{0}$ \\
\hline $\mathrm{GeV}^{2}$ & & & & $\mathrm{GeV}$ \\
0.1826 & 514 & $1.15 \cdot 10^{5}$ & $1.3 \cdot 10^{4}$ & -0.628 \\
0.25 & 98 & $6.2 \cdot 10^{3}$ & $2 \cdot 10^{4}$ & -0.791 \\
0.26 & 67 & $2.2 \cdot 10^{4}$ & $1.6 \cdot 10^{4}$ & -0.813 \\
0.27 & 43.6 & $3 \cdot 10^{4}$ & $2.9 \cdot 10^{3}$ & -0.835 \\
0.29 & 12.6 & $7 \cdot 10^{4}$ & $8.5 \cdot 10^{3}$ & -0.878 \\
0.305 & 1.8 & $1.36 \cdot 10^{5}$ & $4.3 \cdot 10^{4}$ & -0.908 \\
\hline
\end{tabular}

The value of $V_{0}$ reflects the fact that the potential is the Fourier-transform of the scattering amplitude, $V_{0}$ being the constant of the interaction. Gromes [21] evaluated this constant for a linear confinement and obtained the value of $V_{0}$

$$
V_{0} \simeq-2 \sqrt{k} \cdot \mathrm{e}^{-(\gamma-0.5)}
$$

where $k$ is a string tension, $\gamma=0.57721 \ldots$ is the Euler-MacLoraint constant. According to our values $k=0.18-0.305 \mathrm{GeV}^{2}$. And according to (23), it has to vary within the limits $V_{0}=-(0.77 \div 1.02) \mathrm{GeV}$, which is quite close to the values cited in table 2 . Let us stress that the set values are not colour-dependent, which reduces the number of adjustable parameters.

The results of the calculation, together with the experimental data, are shown in tables (3-6). The experimental values were taken from [22]. For choosing the parameters the minimum of the $\chi^{2}$-criterion was used, with the definition of $\chi^{2}$ given in [18]. In this definition $\mathbf{N}$ is the number of meson masses, $\mathbf{n}$ is the number of parameters (in our case two parameters, namely, a confinement parameter and $\left.V_{0}\right), \Delta$ is an experimental error in the definition of experimental mass $M_{\mathrm{EXP}}$ of a 
two-quark system [22]. Since $V_{0}$ is chosen to match the experimental value of the ground-state mass, we are actually left only with one adjustable parameter $A$ (or $\mathrm{k}$ ). Since we do not include LS-forces, we have to take the average center of gravity (COG) value of P-resonances which is calculated according to the formula:

$$
M_{\mathrm{COG}}=\left(\sum_{j}(2 j+1) \cdot M_{j}\right) /\left(\sum_{j}(2 j+1)\right) .
$$

As one can see from both the radial and orbital excitation calculation, the variant which incorporates in one or another way the relativistic kinematics gives a better description of the Regge trajectories which are believed to be linear in $l$ for $M^{2}$.

It is well known that even in a non-relativistic limit one can obtain a good description of $u \bar{u}-d \bar{d}-$ systems on account of spoiling the $c \bar{c}$ of $b \bar{b}$-description.

Indeed, in table 2 we show $\chi^{2}$ obtained for the Eichten parameters. Due to a high precision of defining $\mathrm{J} / \Psi$ and $\Upsilon$-mesons, large $\chi^{2}$ are considered good for these mesons and bad for a $\rho$-meson trajectory. On the other hand, the nonrelativistic Badalyan [23] results are good for $u \bar{u}$, but bad for $J / \Psi$. Fabre [24], in order to obtain good results for light quarkonium, had to change the potential itself. Instead, using our way or incorporating relativistic kinematics we obtain good results for all the data. Table 2 demonstrates this statement. Table 3 contains a comparison of different potentials. The discussion concerning the choice of the potentials is given in [18]. We shall choose in what follows the Cornell-potential (7b) which seems to be preferrable, though potential $(7 \mathrm{c})$ is also quite good. The parameters $\alpha_{s}$ here are taken from table 1 . The $u \bar{u}$-data are fantastically good, but $c \overline{\mathrm{c}}$ and $\mathrm{b} \overline{\mathrm{b}}$-data could be better. Therefore, in tables $4-6$ we give the results for $k=0.29$ $\mathrm{GeV}^{2}$. We consider these results the best. It is interesting that the values which give these results are close to those of Lucha and Schöberl [3]. We want to stress that all the above results are obtained by the numerical solution of (10). The last columns in $(4-6)$ are calculated according to (18). With the choice $A=0.071$ $\mathrm{GeV}^{3}, V_{0}=-1.0077 \mathrm{GeV}, m_{\mathrm{u}}=0.33 \mathrm{GeV}, m_{\mathrm{c}}=1.75 \mathrm{GeV}, m_{\mathrm{b}}=5.13 \mathrm{GeV}$ the results are quite comparable with other entries. But still we have to conclude that a pure oscillator potential is too rough to give the final result. A more sophisticated potential is to be taken here too. But it clearly demonstrates that the inclusion of relativistic kinematics is crucial.

We would like to emphasize one interesting feature of relativistic models, namely, that the slope of a linear (or close to linear) Regge trajectory in this case is constant, while in nonlinear models it is neither constant nor linear. The experiments show this slope to be equal to $\sim 1.2 \mathrm{GeV}^{2}$. In our cases it varies from 1.15 to $2.5 \mathrm{GeV}^{2}$. As Tutik et al. [25] have indicated, the Regge trajectories for low-lying states coincide, while for large values of orbital momentum $l$ the screened potential (7c) leads to a limited Regge trajectory in contrast to infinitely rising tragectories for other potentials, like $(7 \mathrm{a})$ or $(7 \mathrm{~b})$. 


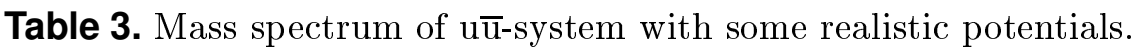

\begin{tabular}{|c|c|c|c|c|c|}
\hline State & designation & $\mathrm{M}_{\mathrm{exp}}$ & $\mathrm{M}_{\mathrm{th}}(7 a)$ & $\mathrm{M}_{\mathrm{th}}(7 b)$ & $\mathrm{M}_{\mathrm{th}}(7 c)$ \\
\hline & & $\mathrm{GeV}$ & $\mathrm{GeV}$ & $\mathrm{GeV}$ & $\mathrm{GeV}$ \\
\hline $1 \mathrm{~S}$ & $\rho 1^{+}\left(1^{--}\right)$ & $0.768 \pm 0.0005$ & 0.77 & 0.77 & 0.77 \\
\hline $1 \mathrm{P}$ & ${ }^{3} \mathrm{P}_{\operatorname{cog}}^{*}$ & $1.262 \pm 0.03$ & 1.26 & 1.296 & 1.3 \\
\hline $2 \mathrm{~S}$ & $\rho 1^{+}\left(1^{--}\right)$ & $1.465 \pm 0.025$ & 1.609 & 1.573 & 1.561 \\
\hline $2 \mathrm{P}$ & $a_{2} 1^{-}\left(2^{++}\right)$ & $1.935 \pm 0.015$ & 2.003 & 1.932 & 1.889 \\
\hline $3 \mathrm{~S}$ & $\rho 1^{+}\left(1^{--}\right)$ & $2.150 \pm 0.01$ & 2.3 & 2.155 & 2.103 \\
\hline $1 \mathrm{D}$ & $\rho_{3} 1^{+}\left(3^{--}\right)$ & $1.691 \pm 0.013$ & 1.665 & 1.689 & 1.687 \\
\hline $2 \mathrm{D}$ & $\rho_{3} 1^{+}\left(3^{--}\right)$ & $2.250 \pm 0.01$ & 2.348 & 2.235 & 2.1845 \\
\hline $1 \mathrm{~F}$ & $a_{4} 1^{-}\left(4^{++}\right)$ & $2.037 \pm 0.036$ & 2.03 & 2.021 & 2.003 \\
\hline $1 \mathrm{G}$ & $\rho_{5} 1^{+}\left(5^{--}\right)$ & $2.350 \pm 0.015$ & 2.366 & 2.312 & 2.275 \\
\hline $1 \mathrm{H}$ & $a_{6} 1^{-}\left(6^{++}\right)$ & $2.450 \pm 0.13$ & 2.685 & 2.576 & 2.514 \\
\hline$\chi^{2}$ & - & - & 53.3 & 1.8 & 19.6 \\
\hline
\end{tabular}

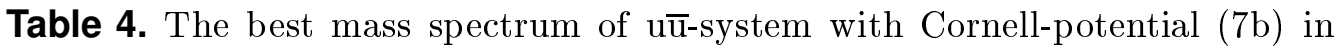
formula (18).

\begin{tabular}{rrlll}
\hline \multirow{5}{*}{ State } & designation & $\mathrm{M}_{\text {exp }}$ & $\mathrm{M}_{\mathrm{th}}(7 b)$ & $\mathrm{M}_{\mathrm{th}}(18)$ \\
& & & \\
\hline & & $\mathrm{GeV}$ & $\mathrm{GeV}$ & $\mathrm{GeV}$ \\
$1 \mathrm{~S}$ & $\rho 1^{+}\left(1^{--}\right)$ & $0.768 \pm 0.0005$ & 0.768 & 0.768 \\
$1 \mathrm{P}$ & ${ }^{3} \mathrm{P}^{*} \mathrm{cog}$ & $1.262 \pm 0.03$ & 1.281 & 1.198 \\
$2 \mathrm{~S}$ & $\rho 1^{+}\left(1^{--}\right)$ & $1.465 \pm 0.025$ & 1.551 & 1.577 \\
$2 \mathrm{P}$ & $a_{2} 1^{-}\left(2^{++}\right)$ & $1.935 \pm 0.015$ & 1.902 & 1.923 \\
$3 \mathrm{~S}$ & $\rho 1^{+}\left(1^{--}\right)$ & $2.150 \pm 0.01$ & 2.12 & 2.244 \\
$1 \mathrm{D}$ & $\rho_{3} 1^{+}\left(3^{--}\right)$ & $1.691 \pm 0.013$ & 1.665 & 1.577 \\
$2 \mathrm{D}$ & $\rho_{3} 1^{+}\left(3^{--}\right)$ & $2.250 \pm 0.01$ & 2.197 & 2.244 \\
$1 \mathrm{~F}$ & $a_{4} 1^{-}\left(4^{++}\right)$ & $2.037 \pm 0.036$ & 1.987 & 1.923 \\
$1 \mathrm{G}$ & $\rho_{5} 1^{+}\left(5^{--}\right)$ & $2.350 \pm 0.015$ & 2.278 & 2.244 \\
$1 \mathrm{H}$ & $a_{6} 1^{-}\left(6^{++}\right)$ & $2.450 \pm 0.13$ & 2.529 & 2.547 \\
$\chi^{2}$ & & - & 12.6 & 35.68 \\
\hline
\end{tabular}


Table 5. The same for cc-system.

\begin{tabular}{rrlll}
\hline & & & & \\
State & designation & $\mathrm{M}_{\text {exp }}$ & $\mathrm{M}_{\mathrm{th}}(7 b)$ & $\mathrm{M}_{\mathrm{th}}(18)$ \\
& & & & \\
\hline & & $\mathrm{GeV}$ & $\mathrm{GeV}$ & $\mathrm{GeV}$ \\
$1 \mathrm{~S}$ & $\mathrm{~J} / \Psi 0^{-}\left(1^{--}\right)$ & $3.096 \pm 0.00009$ & 3.07 & 3.014 \\
$1 \mathrm{P}$ & $\chi_{\mathrm{c} 10^{+}\left(1^{++}\right)}$ & $3.510 \pm 0.00012$ & 3.5118 & 3.331 \\
$1 \mathrm{D}$ & $\Psi ?^{?}\left(1^{--}\right)$ & $3.770 \pm 0.0025$ & 3.837 & 3.632 \\
$2 \mathrm{~S}$ & $\Psi 0^{-}\left(1^{--}\right)$ & $3.688 \pm 0.0001$ & 3.732 & 3.632 \\
$2 \mathrm{D}$ & $\Psi ?^{?}\left(1^{--}\right)$ & $4.159 \pm 0.02$ & 4.296 & 4.195 \\
$3 \mathrm{~S}$ & $\Psi ?^{?}\left(1^{--}\right)$ & $4.040 \pm 0.01$ & 4.227 & 4.195 \\
$3 \mathrm{D}$ & $\Psi ?^{?}\left(1^{--}\right)$ & $4.415 \pm 0.006$ & 4.692 & 4.717 \\
$\chi^{2}$ & & - & $7 \cdot 10^{4}$ & $8.4 \cdot 10^{5}$ \\
\hline
\end{tabular}

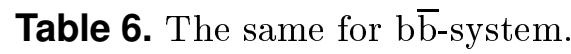

\begin{tabular}{|c|c|c|c|c|}
\hline State & designation & $\mathrm{M}_{\mathrm{exp}}$ & $\mathrm{M}_{\mathrm{th}}(7 b)$ & $\mathrm{M}_{\mathrm{th}}(18)$ \\
\hline & & $\mathrm{GeV}$ & $\mathrm{GeV}$ & $\mathrm{GeV}$ \\
\hline $1 \mathrm{~S}$ & $\Upsilon ?^{?}\left(1^{--}\right)$ & $9.460 \pm 0.00022$ & 9.479 & 9.542 \\
\hline $1 \mathrm{P}$ & ${ }^{3} \mathrm{P}_{\mathrm{cog}}^{*}$ & $9.892 \pm 0.0007$ & 9.883 & 9.741 \\
\hline $2 \mathrm{~S}$ & $\Upsilon ?^{?}\left(1^{--}\right)$ & $10.023 \pm 0.00031$ & 10.037 & 9.932 \\
\hline $2 \mathrm{P}$ & ${ }^{3} \mathrm{P}_{\operatorname{cog}}^{*}$ & $10.268 \pm 0.00057$ & 10.299 & 10.121 \\
\hline $3 \mathrm{~S}$ & $\Upsilon ?^{?}\left(1^{--}\right)$ & $10.355 \pm 0.0005$ & 10.433 & 10.308 \\
\hline $4 \mathrm{~S}$ & $\Upsilon ?^{?}\left(1^{--}\right)$ & $10.580 \pm 0.0035$ & 10.776 & 10.675 \\
\hline $5 \mathrm{~S}$ & $\Upsilon ?^{?}\left(1^{--}\right)$ & $10.865 \pm 0.008$ & 11.062 & 10.034 \\
\hline $6 \mathrm{~S}$ & $\Upsilon ?^{?}\left(1^{--}\right)$ & $11.019 \pm 0.008$ & 11.332 & 11.385 \\
\hline$\chi^{2}$ & & - & $8.5 \cdot 10^{3}$ & $7.4 \cdot 10^{4}$ \\
\hline
\end{tabular}




\section{References}

1. S. Godfrey, M. Isgur, Phys. Rev. D39, 189 (1985).

2. E. Eichten, R. Gottfried, Phys. Rev. D21, 203 (1980).

3. W. Lucha, F. Shöberl, Phys. Rep. 200, 127 (1991).

4. R.P. Gaida, Sov. J. Part. Nucl. 13, 179 (1982).

5. V. Tretyak, V. Shpytko, Ukr. Phys. Journ. 40, 1250 (1995).

6. V. Tretyak, V. Shpytko, J. Nonlin. Math. Phys. 4, No 1-2, 161 (1997).

7. A. Duviryak, V. Tretyak, V. Shpytko, Proc. Workshop Soft Phys. "Hadrons-94" Uzhorod, 353 (1994).

8. I. Todorov, Phys. Rev. D3, 2351 (1971).

9. P. Bogolyubov, Phys. El. Part. Atom. Nuclei. Dubna, 3, 144 (1972).

10. D. Lichtenberg, W. Namgung, J. Wills, E. Predazzi, Zeitschr. Phys. C19, 19-27 (1989).

11. D. B. Lichtenberg, R. Roncaglia, J. Wills, E. Predazzi, M. Rosso, Zeitshr. Phys. C46, 75 (1990).

12. D.B. Lichtenberg, E. Predazzi, R. Roncaglia, C. Rosseti, Zeitshr. Phys. C40, 357 (1988).

13. W. Lucha, F. Shöberl, HEPHY-PUB 611/94 UWThPh-1994-45.

14. I. Haysak, V. Lengyel, A. Shpenik, S. Chalupka, M. Salak, Ukr. Phys. Journ. 41, 370 (1996), see also Proc. "Hadron Structure-94" Conference, Kosice, 219 (1994).

15. S. Deoghuria, S. Chakrabarty, J. Phys. G: Nucl. Phys. 16, 1825 (1990).

16. V. Kukulin, G. Loyola, M. Moshinsky, Phys. Lett. A158, 19 (1991).

17. M. Moshinsky, A. Szezpaniak, J. Phys. A, 22 (1989).

18. V. Lengyel, V. Rubish, Yu. Fekete, S. Chalupka, M. Salak, Journ. Phys. Studies 2, 38 (1998); see also Proc. Int. Conf. "El-100", Uzhgorod, 182 (1997).

19. S. Ishida, M. Oda, Nuovo Cim. A107, 2510 (1994).

20. S. Chalupka, V. Lengyel, M. Salak, Czech. J. Phys. 44, 104 (1994).

21. D. Gromes, Z. Phys. C11, 147 (1981); 14, 94(E) (1982).

22. Part. Data Group, Phys. Rev. D, 1 (1996).

23. A. Badalyan, D. Pariysky, D.Kitoroage, Sov. Journ. Nucl. Phys. 46, 226 (1987).

24. M. Fabre de la Ripelle, Phys. Lett. B205, 97 (1987).

25. A.V. Kholodkov, F. Paccanoni, S.S. Stepanov, and R.S. Tutik, J. Phys. C18, 985 (1992). 


\title{
Роль релятивістичної кінематики у описі двокваркових систем
}

\author{
В. Лендьел ${ }^{1}$, В. Рубіш ${ }^{1}$, Ю. Фекете ${ }^{1}$, С.Халупка ${ }^{2}$, \\ М. Салак ${ }^{3}$
}

1 Ужгородський державний університет, кафедра теоретичної фізики, вул. Волошина, 32, Ужгород, UA 294000, Україна

2 Університет ім. Шафарика, кафедра теоретичної фізики та геофізики,

вул. Мойзесова, 16, Кошіце, 041 54, Словацька республіка

3 Пряшівський університет, кафедра фізики,

вул. 17 грудня, Пряшів, 080 09, Словацька республіка

Отримано 17 лютого 1998 р.

Зроблено спробу врахувати релятивістичну кінематику у описі легких кваркових систем. Багатообіцяючим виглядає підхід до такого врахування, запропонований Р. Ґайдою та його співробітниками. Порівняння результатів з експериментальними даними, що стосуються спектру мас бозонів, показує, що цей підхід знаходиться серед кращих теоретичних інтерпретацій даних.

Ключові слова: легкі кварки, потенціяльна модель, рівняння Дірака, мезони

PACS: $05.20 .-y, 03.30 .+p$ 
